\title{
Optical properties of the iron-pnictide analog $\mathrm{BaMn}_{2} \mathrm{As}_{2}$
}

\author{
A. Antal, ${ }^{1,2}$ T. Knoblauch, ${ }^{1}$ Y. Singh, ${ }^{3}$ P. Gegenwart, ${ }^{3}$ D. Wu,, , 6 and M. Dressel ${ }^{1}$ \\ ${ }^{1}$ 1. Physikalisches Institut, Universität Stuttgart, Pfaffenwaldring 57, 70550 Stuttgart, Germany \\ ${ }^{2}$ Institute of Physics, Budapest University of Technology and Economics and Condensed Matter Research Group, \\ Hungarian Academy of Sciences, 1521 Budapest, Hungary \\ ${ }^{3}$ I. Physikalisches Institut, Georg-August-Universität Göttingen, 37077 Göttingen, Germany
}

(Dated: November 8, 2018)

\begin{abstract}
We have investigated the infrared and Raman optical properties of $\mathrm{BaMn}_{2} \mathrm{As}_{2}$ in the $a b$-plane and along the $c$-axis. The most prominent features in the infrared spectra are the $E_{u}$ and $A_{2 u}$ phonon modes which show clear TO-LO splitting from the energy loss function analysis. All the phonon features we observed in infrared and Raman spectra are consistent with the calculated values. Compared to the iron-pnictide analog $A \mathrm{Fe}_{2} \mathrm{As}_{2}$, this compound is much more two-dimensional in its electronic properties. For $E \| c$-axis, the overall infrared reflectivity is insulating like. Within the $a b$-plane the material exhibits a semiconducting behavior. An energy gap $2 \Delta=48 \mathrm{meV}$ can be clearly identified below room temperature.
\end{abstract}

PACS numbers: $74.25 . \mathrm{Gz}, 74.70 . \mathrm{Xa}, 74.25 . \mathrm{Jb}, 74.20 . \mathrm{Rp}$

\section{INTRODUCTION}

The discovery of superconductivity in layered LaFeAsO ${ }_{0.9} \mathrm{~F}_{0.1}$ four years ago has brought a new trend to synthesize and study new materials which are analogous to this compound $\stackrel{1,2}{ }$ By now, most of the interest is focussed on the materials as $A M_{2} X_{2}$ "122"-type, $A \mathrm{Fe} X$ " 111 "-type and Fe $X$ " 11 "-type which have high superconducting transition temperature $T_{c}$ and can be obtained as high-quality single crystals. Only few studies have been reported on low $T_{c}$ pnictide compounds or those that do not show superconductivity at all $\stackrel{2-5}{-5}^{-}$Among those is $\mathrm{BaMn}_{2} \mathrm{As}_{2}$, that has the same tetragonal $\mathrm{ThCr}_{2} \mathrm{Si}_{2}$-type structure as $M \mathrm{Fe}_{2} \mathrm{As}_{2}$ at room temperature and undergoes no structural transition upon cooling.$\frac{5}{5}$ The antiferromagnetic order appears at $T_{N}=625 \mathrm{~K}$; i.e. at much higher temperature than for the $M \mathrm{Fe}_{2} \mathrm{As}_{2}$ compounds. ${ }^{6}$ While most of the $A M_{2} X_{2}$ parent compounds are metallic, $\mathrm{BaMn}_{2} \mathrm{As}_{2}$ is a semiconductor with a small band gap $\Delta \approx 27 \mathrm{meV}$ according to $d c$-resistivity measurement $\underline{5}$ An et al. suggested that the difference in magnetic and electronic properties compared to those of $\mathrm{MFe}_{2} \mathrm{As}_{2}$ is due to the strong Hund's coupling, the stability of the half-filled $d$-shell of the $\mathrm{Mn}^{2+}$ ( $d^{5}$ ion) and strong spin-dependent Mn-As hybridization $\underline{\underline{7}}$

In this paper, we present mainly the infrared studies on $\mathrm{BaMn}_{2} \mathrm{As}_{2}$ single crystals with the electric field polarized within the $a b$-plane and parallel to the $c$-axis. The sample with a size of $5 \times 5 \times 1 \mathrm{~mm}^{3}$ was grown out of Sn fluxes. The magnetic, transport, and thermal properties of our material have been reported recently ${ }^{5,6}$ For the infrared reflectance measurements, we cleaved the crystal within the $a b$-plane and finely polished the $a c$-plane in order to get shiny surfaces. The temperature dependent reflectivity spectra were measured in a wide frequency range from 40 to $37000 \mathrm{~cm}^{-1}$ using Fourier-transform infrared spectrometers $\left(40-15000 \mathrm{~cm}^{-1}\right)$ and a variable-angle spectroscopic ellipsometer $\left(6000-37000 \mathrm{~cm}^{-1}\right.$, restricted to room temperature). Raman spectra were measured using Jobin-Yvon T64000 spectrometer equipped with a microscope at room temperature. We used $514.5 \mathrm{~nm}$ Ar-laser excitation line with intensity below $1 \mathrm{~mW}$. Spectra were measured in $(a, a)$ and $(b, b)$ polarizations. Several infrared and Raman phonon modes are assigned and compared to the calculated values.

\section{EXPERIMENTAL RESULT AND DISCUSSION}

In the inset of Fig. 11, we plot the room temperature reflectivity for both $a b$-plane and $c$-axis polarization in the entire measured frequency range $\left(40\right.$ to $\left.37000 \mathrm{~cm}^{-1}\right)$. As approaching the zero frequency, $R_{a b}$ shows an upturn towards unity reflectance while $R_{c}$ tends to keep a constant value of approximately $40 \%$. The rather low overall reflectivity $(\sim 30 \%)$ indicates that $\mathrm{BaMn}_{2} \mathrm{As}_{2}$ is a rather bad metal or a semiconductor. Besides the pronounced phonon vibrations in far-infrared region which we will discuss in detail later in this paper, two interband transition can be identified at approximately $7000 \mathrm{~cm}^{-1}$ $(0.87 \mathrm{eV})$ and $26000 \mathrm{~cm}^{-1}(3.2 \mathrm{eV}) \stackrel{8.9}{9}$ The temperature dependence of the reflectivity spectra is displayed in Fig. 1(a) and (b). In the $a b$-plane, the reflectivity below $700 \mathrm{~cm}^{-1}$ increases with decreasing temperature, indicating a metallic behavior. This is consistent with the $d c$-resistivity measurement reported in Ref. 5. Only a very small temperature dependence is observed along the $c$-axis due to the less conducting property.

\section{A. Phonon vibrations}

Due to the low reflection and weak absorption of Ba$\mathrm{Mn}_{2} \mathrm{As}_{2}$ in the $a b$-polarization, a small fraction (about $5 \%$ ) of the far-mid infrared light was found to be transmitted through the sample during the quality-check mea- 


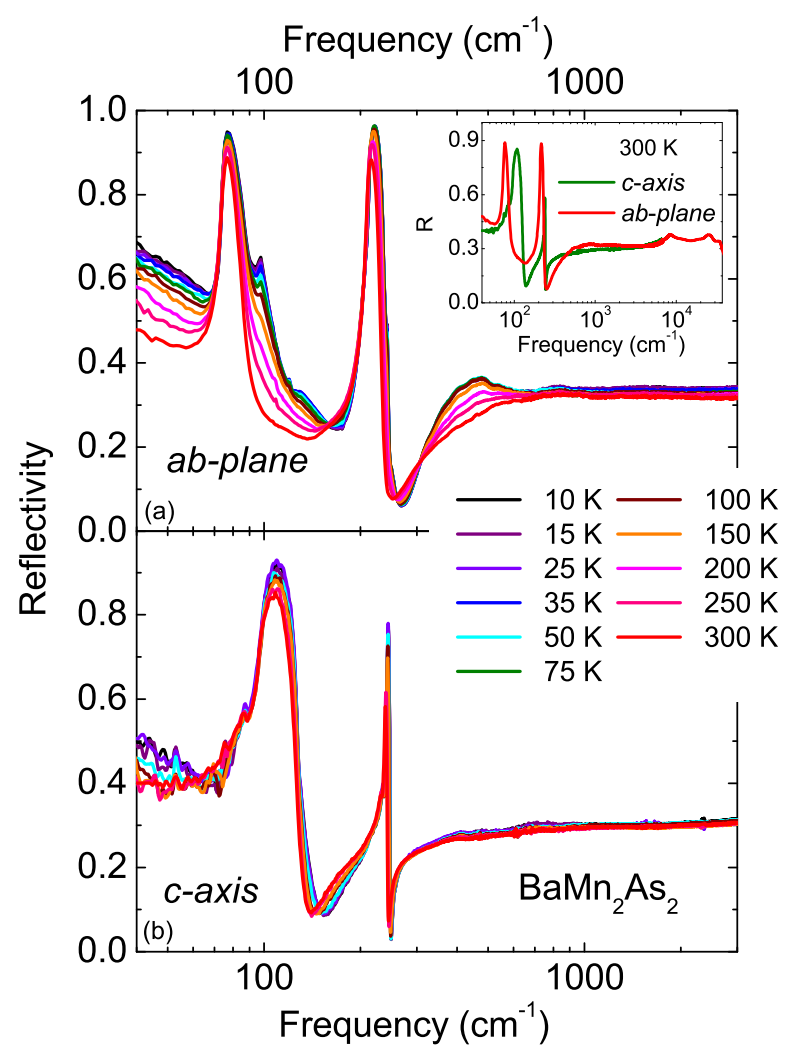

FIG. 1: (Color online) Temperature dependent optical reflectivity of $\mathrm{BaMn}_{2} \mathrm{As}_{2}$ measured in (a) the $a b$-plane and (b) along the $c$-direction. The inset shows the room-temperature reflectivity in the whole measured range $\left(40-37000 \mathrm{~cm}^{-1}\right)$.

TABLE I: Comparison of the phonon mode frequencies (in $\mathrm{cm}^{-1}$ ) found in different $A M_{2} X_{2}$ compounds with the resonance frequencies of $\mathrm{BaMn}_{2} \mathrm{As}_{2}$.

\begin{tabular}{|l|c|c|c|c|c|}
\hline \hline Mode & $\mathrm{CaFe}_{2} \mathrm{As}_{2}$ & $\mathrm{SrFe}_{2} \mathrm{As}_{2}$ & $\mathrm{BaFe}_{2} \mathrm{As}_{2}$ & $\mathrm{EuFe}_{2} \mathrm{As}_{2}$ & $\mathrm{BaMn}_{2} \mathrm{As}_{2}$ \\
\hline $\mathrm{A}_{1 g}$ & 189 & 182 & & & 174 \\
$\mathrm{~B}_{1 g}$ & 211 & 204 & 209 & & \\
$\mathrm{E}_{g}$ & & 114 & 124 & & \\
$\mathrm{E}_{g}$ & & 264 & 264 & & \\
\hline $\mathrm{A}_{2 u}$ & & & & & $96 / 131$ \\
$\mathrm{~A}_{2 u}$ & & & & & $230 / 243$ \\
$\mathrm{E}_{u}$ & & & 94 & & $74 / 92$ \\
$\mathrm{E}_{u}$ & & & 253,254 & 260 & $210 / 238$ \\
\hline Ref. & 12 & 13 & $14-16$ & 8 & this work \\
\hline \hline
\end{tabular}

surement. This prevents us from a correct evaluation of conductivity spectra as commonly done by a Kramers-Kronig analysis 10 Nevertheless, all the pronounced phonon vibration modes show Lorentzian like shapes and thus are applicable for extracting the physics behind. According to the tetragonal $\mathrm{ThCr}_{2} \mathrm{Si}_{2}$-type structure (with space group $\mathrm{I} / \mathrm{mmm}$ ) in which the

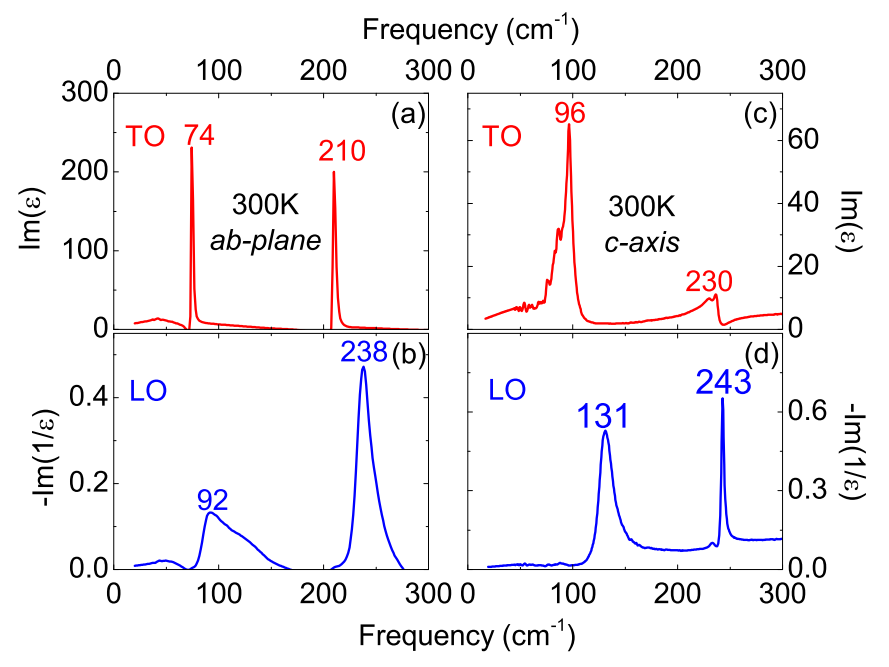

FIG. 2: (Color online) Imaginary part of the dielectric function $\operatorname{Im}(\epsilon)$ and $-\operatorname{Im}(1 / \epsilon)$ at $300 \mathrm{~K}$ for $\mathrm{BaMn}_{2} \mathrm{As}_{2}$ single crystal with $(\mathrm{a}, \mathrm{b}) \mathrm{E} \| \mathrm{ab}$ and $(\mathrm{c}, \mathrm{d}) \mathrm{E} \| \mathrm{c}$. The frequencies of transverse and longitudinal optical modes are respectively identified as labeled in each panel. ${ }^{18}$

$A M_{2} X_{2}$ usually crystallizes, a group theoretical analysis of the phonon modes in $\mathrm{BaMn}_{2} \mathrm{As}_{2}$ yields $\mathrm{A}_{1 g}+\mathrm{B}_{1 g}+2 \mathrm{E}_{g}$ Raman-active, $2 \mathrm{~A}_{2 u}+2 \mathrm{E}_{u}$ infrared-active and $\mathrm{A}_{2 u}+\mathrm{E}_{u}$ acoustic optical zone center phonons, where $\mathrm{A} / \mathrm{B}$ and E modes correspond to an atomic motion perpendicular

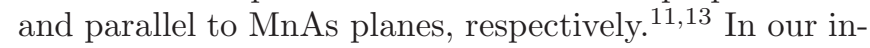
frared spectra, however interestingly, we observe at least four vibration modes both in ab-plane and along the caxis, as shown in Fig. 1. Thus we extract the imaginary part of the dielectric function $\operatorname{Im}(\epsilon)$ and $-\operatorname{Im}(1 / \epsilon)$ (known as the energy loss function) to identify the possible split transverse optical (TO) and longitudinal optical (LO) modes, respectively $\stackrel{17}{\underline{17}}$ According to Fig. 2 , we can directly label the TO/LO energies to be $74 / 92$ $\mathrm{cm}^{-1}$ and $210 / 238 \mathrm{~cm}^{-1}$ for two $\mathrm{E}_{u}$ modes, 96/131 $\mathrm{cm}^{-1}$ and $230 / 243 \mathrm{~cm}^{-1}$ for two $\mathrm{A}_{2 u}$ modes.

In Fig. 3 we show a room-temperature XX $\left(\mathrm{A}_{1 g}\right.$ and $\mathrm{B}_{1 g}$ modes are allowed) Raman spectrum of $\mathrm{BaMn}_{2^{-}}$ $\mathrm{As}_{2}$ as obtained in $(\mathrm{a}, \mathrm{a})$ and $(\mathrm{b}, \mathrm{b})$ polarizations. A pronounced peak can be observed at $174 \mathrm{~cm}^{-1}$. We refer this contribution to the $\mathrm{A}_{1 g}$ mode which is corresponding to the As-As displacement along c-axis (see also in Fig. (4).

In Table.I a comparison of phonon modes frequencies found in $\mathrm{BaMn}_{2} \mathrm{As}_{2}$ and several $A \mathrm{Fe}_{2} \mathrm{As}_{2}(A=\mathrm{Ca}, \mathrm{Ba}$, $\mathrm{Sr}$ and $\mathrm{Eu}$ ) compounds is shown. Due to the differences in lattice constant, atomic mass, and possible magnetic interactions (especially for $\mathrm{EuFe}_{2} \mathrm{As}_{2}$ and $\mathrm{BaMn}_{2} \mathrm{As}_{2}$ ), the vibration frequencies for the same phonon mode are slightly different in these materials.

To compare with theoretical values, we also carried out phonon calculations (in the nonmagnetic state (NM)) on the density-functional perturbation theory level within the plane-wave method with the generalized gradient approximation (GGA) of Perdew-Burke-Enzerhof $\stackrel{19,20}{=}$ We 


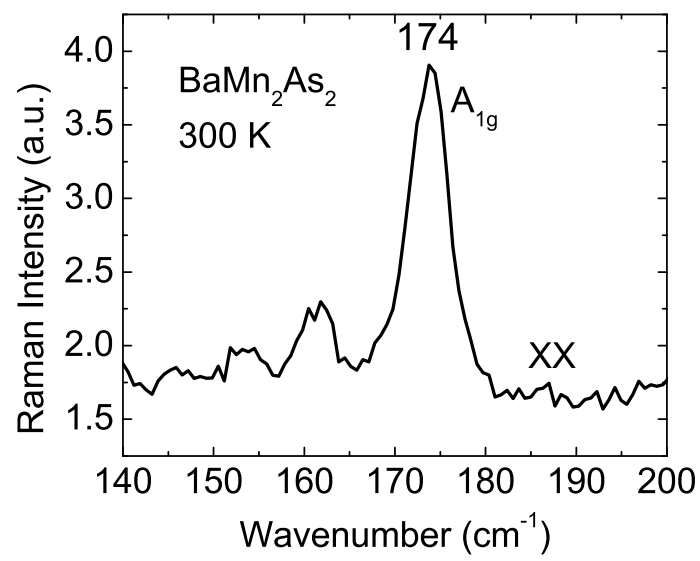

FIG. 3: (Color online) XX-polarized Raman spectrum of Ba$\mathrm{Mn}_{2} \mathrm{As}_{2}$ at $300 \mathrm{~K}$. The peak at $174 \mathrm{~cm}^{-1}$ is assigned as $\mathrm{A}_{1 \mathrm{~g}}$ mode.

used the quantum espresso package for the electronic structure calculation with the implemented ultrasoft plane-wave basis set. $\underline{\underline{21}}$ The band occupation was fixed and the literature lattice parameters has been used ${ }^{22}$ Only the atomic positions in the unit cell were optimized. The Brillouin zone is sampled in the $\mathbf{k}$ space with a uni-

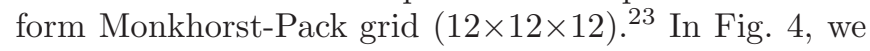
display the calculated atomic transverse optical displacements and the measured TO/LO frequencies for all zone center phonons. Note that, in our calculations only a small LO-TO splitting of less than $1 \mathrm{~cm}^{-1}$ could be observed. The reason for such a small splitting and the discrepancy in the frequencies bases mainly on the nonmagnetic state revealing no band gap. An et al $l^{\underline{7}}$ show by using density functional theory that the ground state of $\mathrm{BaMn}_{2} \mathrm{As}_{2}$ is antiferromagnetic (AFM) with a small bandgap of about $0.2 \mathrm{eV}$ in the case of GGA calculations. Recent $a b$ inito calculations of the phonon spectrum in the " 122 " -family including the magnetic interaction lead to a better agreement of the phonon spectrum. $24-26$ The difference in the mode frequencies between the NM and the AFM state can exceed $40 \mathrm{~cm}^{-1} \underline{27}$ It would be desirable to conduct further calculations including the AFM state which should lead to a small band gap and consequently a bigger LO-TO splitting $?$

In Fig. 5. no change in the phonon shape is observed for these modes due to the absence of structural transition upon cooling. But the $\mathrm{E}_{u}$ mode with higher vibrational frequency is enhanced in intensity and continuously shift to higher frequencies. Recent neutron diffraction measurement on the thermal contraction revealed that by cooling from $300 \mathrm{~K}$ to $10 \mathrm{~K}, d_{\mathrm{Mn}-\mathrm{As}}$ distance decrease

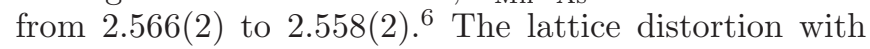
reducing bond lengths enhances the electronic interactions between atoms and therefore the phonon vibrating energy increases - consistent with the observed blue shift of this $\mathrm{E}_{u}$ mode in our spectra.
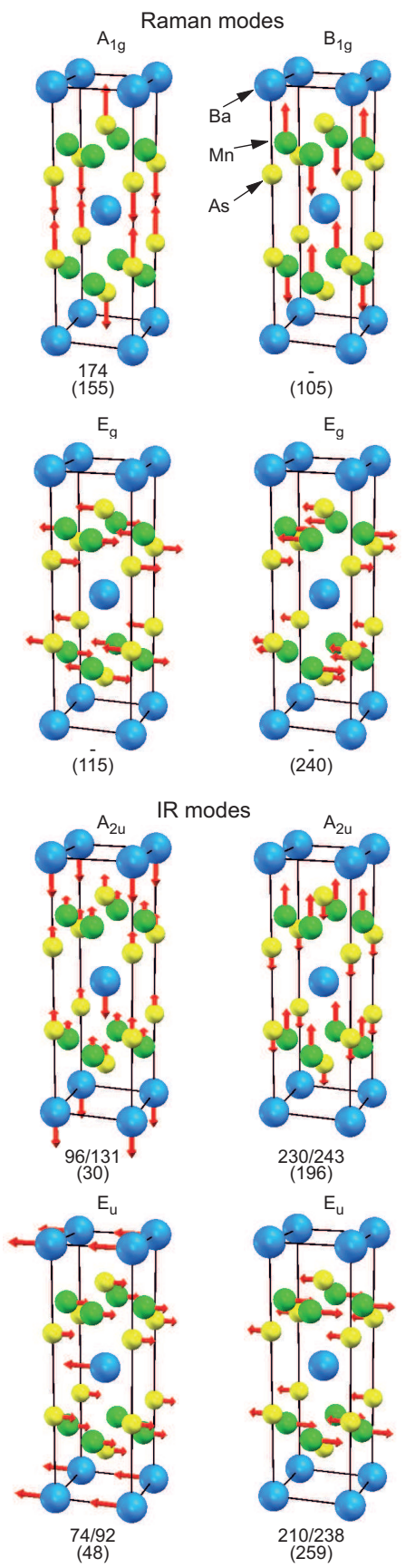

FIG. 4: (Color online) Calculated atomic displacements and $\mathrm{TO} / \mathrm{LO}$ frequencies in $\mathrm{cm}^{-1}$ for zone center phonons. Calculated frequencies are shown in brackets.

\section{B. Energy gap}

There is an extra broad hump at the frequency around $400 \mathrm{~cm}^{-1}$ in the in-plane reflectivity spectra, as shown in Fig.1(a). According to the phonon calculations, we can exclude it to be a phonon mode. As the optical conductivity spectra of $\mathrm{BaMn}_{2} \mathrm{As}_{2}$ are phonon-rich ones, we will here analyze the optical data by looking at the relative change of the conductivity $\Delta \sigma_{1}(\omega, T)=\sigma_{1}(\omega, T)-$ 


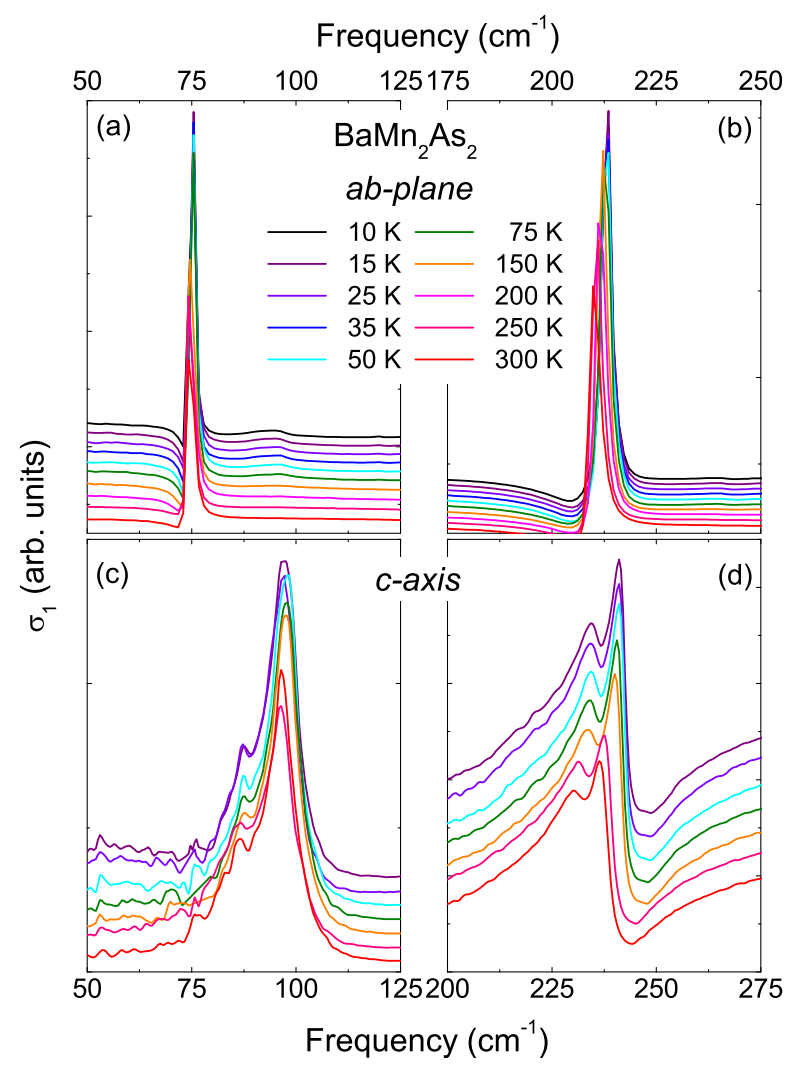

FIG. 5: (Color online) Temperature development of conductivity in the region of phonon modes of $\mathrm{BaMn}_{2} \mathrm{As}_{2}$.

$\sigma_{1}\left(\omega, T_{0}=300 \mathrm{~K}\right)$, as plotted Fig. 6, to get rid of the strong phonon oscillations and see basically only the electronic background and consequently the spectral weight redistribution upon varying the temperature. As seen in Fig. 6] around $300 \mathrm{~cm}^{-1} \Delta \sigma_{1}=\sigma_{1}(\omega, T)-\sigma_{1}(\omega, 300 \mathrm{~K})$ drops to negative values with decreasing temperature, then it increases at higher frequency and crosses zero at $440 \mathrm{~cm}^{-1}$ indicating that the spectral weight redistributes from low to high energies. Such a spectral weight rearrangement is the typical signature for an energy gap formation. From the minimum of $\Delta \sigma_{1}(10 \mathrm{~K})$ we estimate

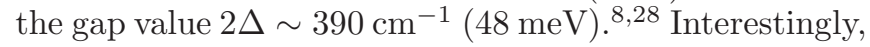
Singh et al. recently performed a gap analysis on the $d c$-conductivity curve of this material. ${ }^{\frac{5}{5}}$ There two linear ranges were found in the $\ln \{\sigma(T)\}$, and according to the expression $\ln \{\sigma\}=A-\Delta / k_{B} T$ two activation energies $\Delta=27 \mathrm{meV}$ and $\Delta=6.5 \mathrm{meV}$ were derived respectively. The gap value we yield from our optical experiments is in accord with the bigger one ${ }^{29}$

\section{Anisotropy}

The $c$-axis conductivity (Fig. 7) varies only slightly with temperatures. When $\omega \rightarrow 0 \mathrm{~cm}^{-1}$, no Drude like behavior is observed and $\sigma_{1}(\omega \rightarrow 0)$ is quite low, indicating that the material is insulating along the $c$-axis.

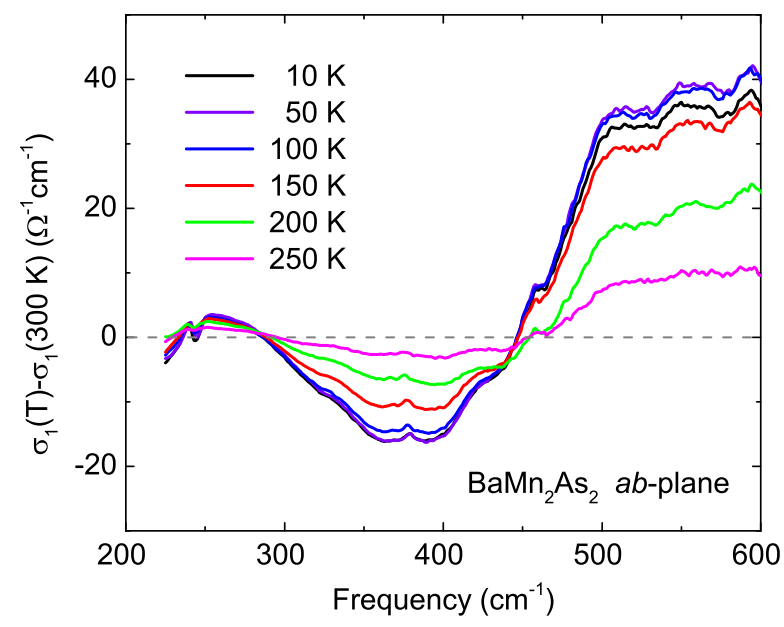

FIG. 6: (Color online) $\Delta \sigma_{1}(\omega, T)=\sigma_{1}(\omega, T)-\sigma_{1}(\omega, 300 \mathrm{~K})$ at selected temperatures.

No transfer of spectral weight is induced by the gap opening. In contrast to iron-pnictides $\mathrm{MFe}_{2} \mathrm{As}_{2}$, the present compound $\mathrm{BaMn}_{2} \mathrm{As}_{2}$ reveals distinctively different electronic properties for the in-plane and out-of-plane directions, i.e. the material is more two-dimensional. In previous optical studies on $\mathrm{BaFe}_{2} \mathrm{As}_{2}$ by Wang and collaborators, 30 the metallic responses were found for both $E \| a b$ and $E \| c$ with an anisotropy-factor about three. 31 While two spin-density-wave gaps were revealed in the $a b$-plane, one of them also opens along the $c$-axis, giving evidence for a three-dimensional Fermi surface in the electronic structure 30 Furthermore, the low carrier mobility in $\mathrm{BaMn}_{2} \mathrm{As}_{2}$ infers more localized charge carriers, in the contrast to the itinerant nature of the electronic properties of $M \mathrm{Fe}_{2} \mathrm{As}_{2}$. The reason is the antiferromagnetic arrangement of the $\mathrm{Mn}^{2+}$ spins both in the $a b$-plane and along the $c$-axis (G-type antiferromagnetic order), where at low temperature the electron hopping between nearest neighbor sites with opposite spin is suppressed and a strong scattering is induced by the spin disorder at high temperature $\underline{\underline{7}}$

\section{SUMMARY}

In summary, the optical properties of iron-pnictide analog $\mathrm{BaMn}_{2} \mathrm{As}_{2}$ have been studied in detail using light polarized in the $a b$-plane and along the $c$-axis. Within the $a b$-plane, the material behaves like a poor metal or semiconductor. An energy gap can be defined at $2 \Delta=48 \mathrm{meV}$. The $c$-axis spectra show a typical insulating behavior overall and no energy gap is found. This indicates that the $\mathrm{BaMn}_{2} \mathrm{As}_{2}$ is a two-dimensional electron system rather than a quasi three-dimensional, as the Fe-pnictides are considered to be. The $E_{u}$ and $A_{2 u}$ phonon modes have clear TO/LO splitting with frequencies differences of $10-40 \mathrm{~cm}^{-1}$. A systematic comparison between experimental results and theoretical calculations 


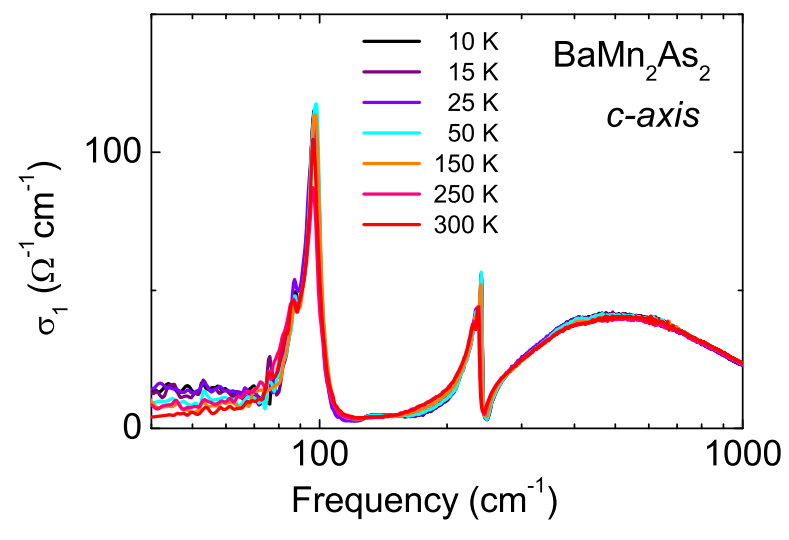

FIG. 7: (Color online) c-axis conductivity of $\mathrm{BaMn}_{2} \mathrm{As}_{2}$ as a function of frequency from 40 to $1000 \mathrm{~cm}^{-1}$ measured at selected temperatures. are present for all the infrared and Raman modes.

\section{Acknowledgments}

We thank B. Gorshunov and V. I. Torgashev for very helpful discussions. We appreciate N. Drichko at Johns Hopkins University, Baltimore MD, for performing the Raman measurement. The contributions of J. Braun and D. Rausch to the experiments are appreciated. D.W acknowledges her fellowship by the Alexander von Humboldt Foundation. A.A. acknowledges the support of the German Academic Exchange Service (DAAD) and also Hungarian National Research Fund OTKA NN76727. T.K. acknowledges his scholarship by the Carl-ZeissStiftung. Part of the work was funded by the Deutsche Forschungsgemeinschaft (DFG).
* Electronic address: dan.wu@pil.physik.uni-stuttgart.de

1 Y. Kamihara, T. Watanabe, M. Hirano, and H. Hosono, J. Am. Chem. Soc. 130, 3296 (2008).

2 D. C. Johnston, Adv. Phys. 59, 803 (2010).

${ }^{3}$ F. Ronning, N. Kurita, E. D. Bauer, B. L. Scott, T. Park, T. Klimczuk, R. Movshovich, and J. D. Thompson, J. Phys.:Condens. Matter 20, 3422033 (2008)

${ }^{4}$ R. Nath, V. O. Garlea, A. I. Goldman, D. C. Johnston, Phys. Rev. B 81, 224513 (2010).

5 Y. Singh, A. Ellern, and D. C. Johnston, Phys. Rev. B 79, 094519 (2009).

6 Y. Singh, M. A. Green, Q. Huang, A. Kreyssig, R. J. McQueeney, D. C. Johnston, and A. I. Goldman, Phys. Rev. B 80, 100403 (2009)

7 J. An, A. S. Sefat, D. J. Singh, and M.-H. Du, Phys. Rev. B 79, 075120 (2009).

${ }^{8}$ D. Wu, N. Barišić, N. Drichko, S. Kaiser, A. Faridian, M. Dressel, S. Jiang, Z. Ren, L. J. Li, G. H. Cao, Z. A. Xu, H. S. Jeevan and P. Gegenwart, Phys. Rev. B 79, 155103 (2009)

${ }^{9}$ G. Li, W. Z. Hu, J. Dong, Z. Li, P. Zheng, G. F. Chen, J. L. Luo, and N. L. Wang, Phys. Rev. Lett. 101, 107004 (2008).

10 M. Dressel and G. Grüner, Electrodynamics of Solids (Cambridge University Press, Cambridge, 2002).

11 E. Kroumova, M. I. Aroyo, J. M. Perez-Mato, A. Kirov, C. Capillas, S. Ivantchev and H. Wondratschek, Phase Transition 76, 155-170 (2003).

12 K.-Y. Choi, D. Wulferding, P. Lemmens, N. Ni, S. L. Bud'ko and P. C. Canfield, Phys. Rev. B 78, 212503 (2008).

13 A. P. Litvinchuk, V. G. Hadjiev, M. N. Iliev, Bing Lv, A. M. Guloy and C. W. Chu, Phys. Rev. B 78, 060503(R) (2008).

14 L. Chauvière, Y. Gallais, M. Cazayous, A. Sacuto, M. A. Méasson, D. Colson and A. Forget, Phys. Rev. B 80, 094504 (2009).

15 A. Akrap, J. J. Tu, L. J. Li, G. H. Cao, Z. A. Xu, and C. C. Homes, Phys. Rev. B 80, 180502 (2009).
16 A. A. Schafgans, B.C. Pursley, A. d. LaForge, A. S. Sefat, D. Mandrus and D. N. Basov, Phys. Rev. B 84, 052501 (2011).

17 P-Y. Yu and M. Cardona, Fundamentals of Semiconductors, Physics and Materials Properties (Springer, 1996).

18 In Fig. 22(a) and (b), there are ranges (for example, 170-210 $\left.\mathrm{cm}^{-1}\right)$ where $\operatorname{Im}(\epsilon)$ shows negative values. This unphysical phenomenon is caused by the transparent property of sample as we discussed at the beginning of II-A section.

19 S. Baroni, S. de Gironcoli, A. Dal Corso and P. Giannozzi, Rev. Mod. Phys. 73, 515-560 (2001).

20 J. P. Perdew, K. Burke, and M. Enzerhof, Phys. Rev. Lett. 77, 3865 (1996).

21 P. Giannozzi et al., J. Phys.: Condens. Matter, 21, 395502, (2009).

22 Y. Singh, M. A. Green, Q. Huang, A. Kreyssig, R. J. McQueeney, D. C. Johnston and A. I. Goldman Phys. Rev. B 80, 100403 (2009).

23 H. J. Monkhorst and J. D. Pack, Phys. Rev. B 13, 5188 (1976).

24 R. Mittal, S. Rols, M. Zibri, Y. Su, H. Schober, L. Chaplot, M. Johnson, M. Tegel, T. Chatterji, S. Matsuishi, H. Hosono, D. Johrendt and Th. Brueckel, Phys. Rev. B 79, 144516 (2009).

25 M. Zbiri, H. Schober, M. R. Johnson, S. Rols, R. Mittal, Y. $\mathrm{Su}, \mathrm{M}$. Rotter and D. Johrendt, Phys. Rev. B 79, 064511 (2009)

26 E. Aktürk and S. Ciraci, Phy. Rev. B 79, 184523 (2009).

27 P. Kumar, A. Bera, D. V. S. Muthu, A. Kumar, U. V. Waghmare, L. Harnagea, C. Hess, S. Wurmehl, S. Singh, B. Bëhner and A. K. Sood, J. Phys.: Condens. Matter 23 (2011) 255403.

28 W. Z. Hu, J. Dong, G. Li, Z. Li, P. Zheng, G. F. Chen, J. L. Luo and N. L. Wang, Phys. Rev. Lett. 101, 257005 (2008).

29 At around $100 \mathrm{~cm}^{-1}$ we observed a similar but very small hump at $10 \mathrm{~K}$ and $50 \mathrm{~K}$. It can be due to the formation of a smaller gap $2 \Delta=12 \mathrm{meV}$. As this gap-like feature only appears below $75 \mathrm{~K}$, it should be referred to the second 
gap $\Delta=6.5 \mathrm{meV}$ measured in $d c$-resistivity by Singh et. al in Ref.5.

30 Z. G. Chen, T. Dong, R. H. Ruan, B. F. Hu, B. Cheng, W. Z. Hu, P. Zheng, Z. Fang, X. Dai, and N. L. Wang, Phys.
Rev. Lett. 105, 097003 (2010).

31 In the case of $\mathrm{EuFe}_{2} \mathrm{As}_{2}$ the anisotropy was about a factor of 8 (Ref. 8). 